\title{
Long-term Clinical Results of Laparoscopic Splenectomy for Surgical Disease of the Spleen: Recent Outcomes
}

\author{
Jeong Eun Seo, M.D., Seog Ki Min, M.D., Hyeon Kook Lee, M.D. \\ Department of Surgery, School of Medicine, Ewha Womans University, Seoul, Korea
}

Purpose: Laparoscopic splenectomy (LS) is one method for treatment of various diseases of the spleen, especially hematological conditions. However, few recent long-term follow-up results have been reported. The purpose of this study is to evaluate the outcome of patients in a single institution who recently underwent $\mathrm{LS}$ and to analyze their long-term follow-up results.

Methods: Of 366 splenectomies, this study was conducted as a retrospective review of 52 patients who underwent LS for treatment of hematological or primary diseases of the spleen from January 1998 to October 2011. The data included age, sex, pathological diagnosis, operative time, postoperative hospital stay, rate to open conversion, perioperative transfusion, morbidity, mortality, and relapse. We analyzed outcomes of variable results through long-term follow-up.

Results: The mean follow-up period was 84 months (range, 4 147 months). The most common indication for LS was im- mune thrombocytopenic purpura (ITP). The median postoperative hospital stay was eight days (range, $3 \sim 28$ days). Mean operative time was 203 minutes (range, 115 475 minutes). Two patients underwent open conversion. Thirty eight patients received perioperative transfusions. The mean spleen weight was $294.9 \mathrm{~g}$ (range, $31 \sim 2,564 \mathrm{~g}$ ). The overall morbidity rate was $5.8 \%$ and one patient experienced relapse. Of the 28 patients with ITP, $89.3 \%$ responded to LS.

Conclusion: LS should be one of the best treatment options regardless of splenomegaly and spleen-associated diseases. In particular, for patients with ITP, LS has shown very effective long-term follow-up results. Therefore, LS should be more actively considered as an early treatment option in surgical disease of the spleen, such as ITP.

Key words: Laparoscopic splenectomy, Hematologic diseases, Immune thrombocytopenic purpura

\section{INTRODUCTION}

Splenectomy is performed for many reasons, particularly for a range of benign and malignant hematological disorders of the spleen such as immune thrombocytopenic purpura (ITP) and hereditary spherocytosis in adult and pediatric patients. ${ }^{1-4}$

By virtue of development of laparoscopic techniques, laparoscopic splenectomy (LS) has become widely used and been established as a safe and effective treatment for various diseases associated with the spleen and is highly recommended even to the area that was indicated for splenectomy but not always performed by laparoscopic approach due to the risk of open conversion. To date, surgical outcomes and follow-up results after LS have been reported in many studies. However, practically most patients who were indicated for LS chose medical treatment first, because the patients, and their families and doctors worried about operative risk including open conversion and

Received October 12, 2013, Revised November 11, 2013,

Accepted November 25, 2013

※ Corresponding author : Seog Ki Min

Department of Surgery, Ewha Womans University Mokdong Hospital, School of Medicine, Ewha Womans University, 1071, Anyangcheon-ro, Yangcheon-gu, Seoul 158-710, Korea

Tel : +82-2-2650-5599, Fax : +82-2-2644-7984

E-mail : mp9666@ewha.ac.kr wound scar. And long-term follow-up results and recent data were insufficient, so they had a limitation to provide the latest information to the doctors in pediatrics, hemato-oncology, and other internal medicine specialties who have treated patients with splenic diseases.

As this important role of surgeon has been gradually insignificant in medicine, we studied patients who recently underwent LS in a single institution and analyzed their long-term follow-up results. Particularly, we intend to investigate the change of results compared to previous studies and to extend the indications of LS. Because if we support the surgical security of LS definitely, medicine doctor can consider LS more actively for early treatment option in surgical disease of the spleen, such as ITP.

\section{MATERIALS AND METHODS}

\section{1) Patients and indications for LS}

Of the total 366 splenectomies that we performed between January 1998 and October 2011 at our clinic, we retrospectively reviewed the 61 patients who underwent LS. We performed LS according to general indications. ${ }^{5}$ Those are mostly benign hematologic conditions including ITP, hereditary spherocytosis, hemolytic anemia caused by erythrocyte enzyme deficiency, and hemoglobinopathies. In addition, there are malignant hema- 
tologic disease such as lymphoma or leukemia, non-hematologic tumors of the spleen, and miscellaneous benign conditions such as splenic cysts, abscess, trauma, and wandering spleen, etc. LS has been reported for most splenic disease and is the preferred method for most situations, barring trauma or cases of massive splenomegaly. When deciding whether to pursue laparoscopic method for splenectomy, certain considerations should be taken into account, such as operative indication, splenic size, and any potential contraindications to laparoscopy. ${ }^{5}$ We excluded cases of open splenectomy from the beginning. Of the 61 patients who underwent LS, concomitant LS with other operations or trauma-related conditions were excluded and finally the medical records and follow-up information were available for 52 patients (Fig. 1). The mean follow-up period was 84 months (range, $4 \sim 147$ months).

\section{2) Data collection}

The data extracted included patient age, sex, pathological diagnosis, duration of operation, postoperative hospital stay, incidence of accessory spleen, open conversion rate, perioperative transfusion requirement, and spleen weight and size. Each patient's platelet count was measured before and after surgery. The peak postoperative platelet count was recorded for each patient. Long-term follow-up data including duration, platelet count, postoperative medical therapy, and complications were obtained from outpatient medical records.

\section{3) Preoperative treatment}

Preoperative surgical planning was aided mostly by computed tomography (CT) imaging especially with regard to splenomegaly and the presence of accessory splenic tissue. Almost all of the pediatric patients were vaccinated 2 weeks before the operation, while in adults, the vaccinations were performed before the operation whenever possible.

\section{4) Operative technique}

Under general anesthesia, the patients were positioned in a right, lateral, semisupine position that was previously described as the "leaning" position. ${ }^{6}$ In the operation, we usually used four trocars. An 11-mm periumbilical port was inserted at the left side of the umbilicus using an open technique and a blunt trocar. The remaining ports were inserted under direct visual support and consisted of the following: one 12-mm trocar approximately $5 \mathrm{~cm}$ inferior to the left subcostal and midclavicular line, one 5-mm trocar in the epigastrium just inferior to the xyphoid process, and one 5-mm trocar at the left lateral flank area. For dissection or ligation of the hilar vessels and short gastric vessels, we used a Harmonic Scalpel (Ethicon Endo-Surgery Inc., Cincinnati, OH, USA) for thin-diameter vessels and an Endo clip (Covidien, Mansfield, MA, USA) or Hem-o-lok clip (Weck Closure Systems, Research Triangle Park, Durham, NC, USA) for thick-diameter vessels. Sometimes a linear stapler (Endo GIA ${ }^{\circledR}$, Covidien, Mansfield, MA, USA) was used for very large vessels that could not be treated with a large clip. Further, when the dissection of the vessels was very difficult to manipulate, we, although rarely, used a linear stapler (Endo GIA ${ }^{\circledR}$, Covidien, Mansfield, MA, USA) to cut the spleen with the pancreatic tail. If the intra-abdominal cavity was large enough and the resected spleen could be placed in the largest laparoscopic pouch, we brought the bag outside the abdomen through the largest incision, most commonly the $12-\mathrm{mm}$ port site. The spleen was then morcellated with ring forceps and removed in fragments. However, if the spleen was huge not to be taken out through the largest incision, we made an additional Pfannenstiel incision to hide the scar.

\section{5) Follow-up}

In the pediatric patients, postoperative follow-up was carried out in the pediatric department. If their postoperative responses

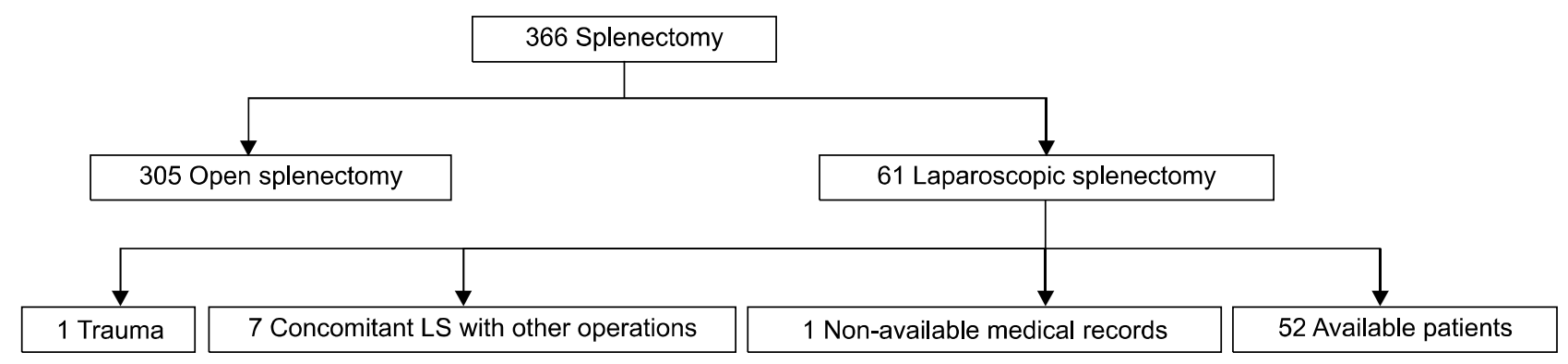

Fig. 1. Patients and indications for LS. 
were good, we provided follow-up only rather than additional treatment. However, if no response was observed, the medical treatment was re-tried. However, splenectomy is usually considered when medical treatment fails, as medical re-treatment is ineffective. The postoperative treatment given to adults is similar to that given to pediatric patients.

The patients were seen regularly in the general surgical outpatient clinic 1 week after discharge and followed at 4 weeks and one year after the operation with a blood test to check for abnormal values including platelet counts. Otherwise, the hematological follow-up varied according to the underlying diagnosis. Imaging studies were not performed routinely for long-term follow-up. We performed abdominal CT follow-up only if there was no response after splenectomy, possibly due to a missed accessory spleen.

\section{6) Definition of response to $\mathrm{LS}$}

In patients with ITP, a response criteria to LS was defined as a rise in platelet count to $50 \times 10^{9} / \mathrm{L}$ without medical treat-

Table 1. The demographic data of patients undergoing successful laparoscopic splenectomy

\begin{tabular}{lc}
\hline \multicolumn{1}{c}{ Characteristics } & Value \\
\hline Male : female $(\mathrm{n}: \mathrm{n})$ & $15: 37$ \\
Pediatrics : adults $(\mathrm{n}: \mathrm{n})$ & $9: 43$ \\
Age (year) & $41.9 \pm 20$ (range, $8 \sim 82)$ \\
\hline
\end{tabular}

Values are presented as mean \pm standard deviation (range).

Table 2. Pathologic diagnosis for laparoscopic splenectomy

\begin{tabular}{lc}
\hline \multicolumn{1}{c}{ Diagnosis } & $\mathrm{n}(\%)$ \\
\hline Benign disease & $49(94.2 \%)$ \\
Immune thrombocytopenic purpura & $28(53.8 \%)$ \\
Hereditary spherocytosis & $7(13.5 \%)$ \\
Infarction & $4(7.7 \%)$ \\
Cavernous hemangioma & $2(3.8 \%)$ \\
Cyst & $2(3.8 \%)$ \\
Abscess & $2(3.8 \%)$ \\
Hereditary hemolytic anemia & $1(1.9 \%)$ \\
Congenital dyserythropoietic anemia & $1(1.9 \%)$ \\
Lymphangioma & $1(1.9 \%)$ \\
Myelodysplastic syndrome & $1(1.9 \%)$ \\
Malignant disease & $3(5.8 \%)$ \\
Lymphoma & $2(3.8 \%)$ \\
Rule out metastasis* & $1(1.9 \%)$
\end{tabular}

*Pathologic diagnosis was not malignant. ment, while no response to LS was defined as no increase in platelet count without medication or an initial rise to $50 \times 10^{9} / \mathrm{L}$ that later decreased to $50 \times 10^{9} / \mathrm{L}$. Relapse was defined as a fall in platelet count to $<50 \times 10^{9} / \mathrm{L} 2$ months after surgery or the need for medical or surgical intervention to maintain the platelet count above this threshold. ${ }^{7}$

\section{7) Statistical analysis}

Statistical analysis was performed using IBM SPSS ver. 19.0 (IBM Co., Armonk, NY, USA). Data are expressed here as median and range. Continuous variables are expressed as mean \pm standard deviation (SD) (range) and compared using Student's $t$ test.

\section{RESULTS}

\section{1) General characteristics}

During the study period, a total of 52 indicated LS were performed in 37 female and 15 male patients (nine pediatric patients, 43 adult patients). The mean patient age was 41.9 years (range, $8 \sim 82$ years) (Table 1 ).

Benign disease was present in 49 of 52 cases (94.2\%); among these, ITP was the most common indication for LS (28 cases; $53.8 \%$ ), followed by hereditary spherocytosis ( 7 cases; $13.5 \%)$. Of the three patients with suspicious for malignant disease, two patients had pathologically confirmed lymphoma after operation (Table 2).

\section{2) Operative results}

The median postoperative hospital stay was 8 days (range, $3 \sim 28$ days). The mean operative time was 203 minutes (range, $115 \sim 475$ minutes). Two patients had an accessory spleen, while another two patients had an open conversion (Table 3).

Thirty-eight patients $(73.1 \%)$ received perioperative transfusions (Table 3); of these, 13 received $\mathrm{RBC}$ transfusions and

Table 3. Operative data

\begin{tabular}{lc}
\hline & Value \\
\hline Operative time (minute) & $203 \pm 83$ (range, 115 475) \\
Postoperative hospital stay (day) & $8.6 \pm 5.5$ (range, 3 28) \\
Incidence of accessory spleen, n (\%) & $2(3.8)$ \\
Incidence of open conversion, $\mathrm{n}(\%)$ & $2(3.8)$ \\
Perioperative transfusion, $\mathrm{n}(\%)$ & $38(73.1)$ \\
Spleen weight $(\mathrm{g})$ & $294.9($ (range, $31 \sim 2,564)$ \\
Maximum spleen size $\left(\mathrm{cm}^{3}\right)$ & $30 \times 22 \times 11$
\end{tabular}

Values are presented as mean \pm standard deviation (range). 
24 patients received any transfusion including platelet constituents. Of the 21 patients who were given a preoperative platelet transfusion, 19 had ITP and the other two had lymphoma. Their preoperative platelet counts were $1,000 \sim 63,000 / \mu 1$.

Mean spleen weight was $294.9 \mathrm{~g}$ (range, $31 \sim 2,564 \mathrm{~g}$ ). The maximum spleen size was $30 \mathrm{~cm}$ in length and $22 \mathrm{~cm}$ in width (Table 3).

\section{3) Outcome and patient status during follow-up}

We experienced postoperative complications in three cases including one patient each with wound hematoma, gastrointestinal bleeding and cerebral infarction, so the overall morbidity rate was $5.8 \%$ (Table 4). Of the three patients, one ITP patient had wound hematoma, and other two patients had non-ITP disease. The last patient, an 82-year-old woman, underwent LS for splenic infarction that was detected when she had been admitted to a hospital due to cerebral infarction about 1 month prior. She had a recurrent acute cerebral infarction on the third postoperative day and was transferred to the neurology department without our problem.

Relapse occurred in one patient with ITP. None of the patients died (Table 4, 5).

For evaluation of long-term follow-up results, in cases of ITP, the platelet count was a significant factor for determining the outcome of LS. The mean preoperative platelet count was $19,300 / \mu 1$, while that during long-term follow-up after LS was increased to $253,600 / \mu 1$. Twenty-five of the 28 patients with ITP $(89.3 \%)$ responded well to the surgery. One patient $(3.6 \%)$ required a second surgery due to disease relapse that was activation of accessory spleen (Table 5). This patient, a 32-year-old woman, had undergone LS for ITP but was diagnosed with a remnant accessory spleen 5 years later. Thus, she underwent an additional laparoscopic accessory splenectomy and was declared recovered during the long-term follow-up. On the other hand, in cases of non-ITP, no relapse was found in long-term follow-up results.

Table 4. Outcome of laparoscopic splenectomy

\begin{tabular}{lc}
\hline & $\mathrm{n}(\%)$ \\
\hline Morbidity & $3(5.8 \%)$ \\
Wound hematoma & 1 \\
Gastrointestinal bleeding & 1 \\
Cerebral infarction & 1 \\
Disease relapse & $1(1.9 \%)$ \\
Mortality & $0(0 \%)$ \\
\hline
\end{tabular}

\section{DISCUSSION}

Since LS was first reported by Delaitre and Maignien in $1991,{ }^{8}$ many surgeons use the laparoscopic approach for the most elective splenectomies. This technique was quickly adopted, as it results in more rapid postoperative recoveries, shorter hospital stays, and lower complication rates than open splenectomy. ${ }^{9}$ However, it still has a few disadvantages including longer operating time and increased risk of intraoperative bleeding due to technical difficulties in securing vascular control at the splenic hilum. ${ }^{10}$ In particular, we have encountered a few cases of massive bleeding due to splenomegaly.

LS is currently the gold standard for several hematologic diseases in which surgical removal of the spleen is indicated. ${ }^{11}$ ITP is an acquired disorder that leads to immune-mediated destruction of platelets and possible inhibition of platelet release from the megakaryocytes. ${ }^{12}$ Patients with platelet counts persistently lower than $30 \times 10^{9} / \mathrm{L}$ are at risk of life-threatening bleeding. The incidence of fatal bleeding was estimated to be $0.02 \sim 0.04$ cases per patient-year, with the highest incidence ( 0.13 cases per patient-year) among patients over 60 years of age. ${ }^{13}$ Therefore, LS is a useful treatment for ITP in this respect.

ITP, the most common autoimmune hematological disorder, is characterized by accelerated antibody-mediated platelet destruction. ${ }^{14,15}$ Splenectomy was the first effective treatment described for ITP and was an established therapeutic modality. Accordingly, ITP is currently the most common indication for splenectomy. Most patients with ITP undergo splenectomy after several years of failed medical treatment. ${ }^{5}$ The splenectomy response rate is reportedly $71 \sim 95 \%$, while the relapse rate is reportedly $4 \sim 12 \%$. $^{5}$

A literature review of 6,942 cases reported before 1996 found a $1.2 \%$ mortality rate from severe infection after splenectomy for ITP. ${ }^{16}$ A recent review of 402 cases of splenectomy for ITP between 1959 and 2002 reported that $1.5 \%$ of all patients developed perioperative infections (most commonly pneumonia). Further, $8 \%$ of patients (one-third of whom had been

Table 5. Long-term follow-up results of the 28 patients with ITP

\begin{tabular}{lc}
\hline Platelet count & \\
$\quad$ Preoperative count & $19,300 / \mu 1$ \\
$\quad$ Postoperative long-term follow-up count & $253,600 / \mu 1$ \\
Response, n (\%) & $25(89.3 \%)$ \\
No response, n (\%) & $3(10.7 \%)$ \\
Relapse, n (\%) & $1(3.6 \%)$ \\
\hline
\end{tabular}


vaccinated) developed infectious complications during follow-up. ${ }^{17}$ In the study of Kang et al., ${ }^{18}$ infections were the most frequent morbidities during the follow-up period. However, in the current study, neither postoperative deaths nor subsequent fatal infections were observed, despite the fact that only five of 28 patients with ITP were vaccinated. The only morbidity in ITP cases was wound hematoma. Other studies of LS for ITP reported an overall $8.4 \%$ major morbidity rate and $0.6 \%$ mortality rate in 127 patients, ${ }^{7}$ and in 23 patients $(16 \%)$ of a total of 147 patients (Table 6). ${ }^{19}$ It has been suggested that preoperative vaccination does not play a significant role in adults prior to undergoing LS, but further research is required to validate this hypothesis.

Our results confirm the excellent hematological outcome of LS for the treatment of chronic ITP. Zheng et al. ${ }^{7}$ reported that patients who responded to LS tended to have a higher platelet count, but no significant difference was seen between the response and no-response groups before surgery $(p=0.070)$. On the other hand, the peak postoperative platelet count was remarkably higher in the response group and was the only independent predictor of hematological outcome $(p<0.001)$. In this study, mean preoperative platelet count was $19,300 / \mu 1$, while that in long-term follow-up was increased to $253,600 / \mu 1$. Of the 28 patients with ITP who were followed up for at least one month, only three $(10.7 \%)$ did not respond to the procedure. After a mean follow-up of 84 months, an overall response rate of $89.3 \%$ was still observed. Only one case of relapse occurred. Other studies reported an $80.3 \%$ long-term response rate of the 127 patients, $^{7}$ an $85 \%$ rate of remission in the total of 147 patients, ${ }^{19}$ and an $88.2 \%$ response rate and $15.2 \%$ relapse rate during the follow-up period in 59 patients (Table 6). ${ }^{18}$ Also, in this study, a single relapsed patient was treated with re-operation by laparoscopic method, so this result must be excellent outcome.

Furthermore, compared to previous studies, this study had some strengths. First, it had a longer follow-up period (mean,

Table 6. Results compared with other studies

\begin{tabular}{lrcrr}
\hline & Morbidity & Mortality & Response & Relapse \\
\hline Zheng CX et al. $^{7}$ & $8.4 \%$ & $0.6 \%$ & $80.3 \%$ & \\
Machado NO et al. $^{10}$ & $16.7 \%$ & & & \\
Kang CM et al. $^{18}$ & $10.9 \%$ & 0 & $88.2 \%$ & $15.2 \%$ \\
Rosen M et al. $^{19}$ & $16 \%$ & $0.68 \%$ & $85 \%$ & $15.0 \%$ \\
Pattenden CJ et al. $^{20}$ & $15 \%$ & 0 & & \\
Bulus H et al. $^{21}$ & $4.8 \%$ & & & \\
This study & $5.8 \%$ & 0 & $89.3 \%$ & $1.9 \%$ \\
\hline
\end{tabular}

84 months) than previous studies: one had a mean of 43.6 months and a range of 9 114 months; ${ }^{7}$ another had a mean of $20 \pm 14$ months; ${ }^{19}$ and a third had a mean of 54 months and range of $12.5 \sim 129$ months. ${ }^{18}$ Second, this study demonstrated lower morbidity and mortality rates. In this study, regardless of diagnosis, the overall morbidity rate was $5.8 \%$. One patient each experienced wound hematoma, gastrointestinal bleeding, and cerebral infarction. Pattenden et al. ${ }^{20}$ reported an overall complication rate of $15 \%$ (Table 6). Further, this study reported a lower incidence of open conversion (two cases; 3.8\%). A review of earlier studies revealed that seven patients $(5 \%),{ }^{19}$ four patients $(6.4 \%),{ }^{21}$ and three patients $(2.1 \%)^{20}$ required conversion to open splenectomy.

LS for splenomegaly remains controversial, as it has traditionally been contra-indicated. Data in the literature have demonstrated an increased risk of hemorrhage, morbidity, and conversion to an open procedure. ${ }^{20}$ However, recent studies have reported that splenomegaly should not be an absolute contra indication to the laparoscopic approach. In the study of Swanson et al., 39 of 217 splenectomies were performed for spleens lager than $20 \mathrm{~cm}$ and demonstrated that hand-assisted LS was a safe and effective technique and a good alternative to open splenectomy. Another study also reported an open conversion rate of only $5.7 \%$ for cases of splenomegaly. ${ }^{20}$ In this study, the mean spleen weight was $294.9 \mathrm{~g}$ (range, $31 \sim 2,564 \mathrm{~g}$ ). The maximum spleen size was $30 \mathrm{~cm}$ in length and $22 \mathrm{~cm}$ in width. The largest spleen found in this study was also removed by laparoscopic method. Open conversion was required in only two cases. In one patient, an 80-year-old woman with ITP that had been treated at the internal medicine department, a gastrointestinal stromal tumor (GIST) was found in the stomach during esophagogastroduodenoscopy. LS was planned first, followed by laparoscopic wedge resection of the GIST by upper gastrointestinal surgeon. The LS was performed successfully, but the stomach GIST was difficult to manipulate because the lesion was placed posterior wall just proximal to the stomach angle, lesser curvature; hence, open conversion was performed. The other case was a 73-year-old man with lymphoma. Because bleeding control failed during the operation, open conversion was conducted. Therefore, true open conversion case was only one.

Thus, although the splenomegaly was severe, a combination of the laparoscopic approach and removal of the specimen through an additional incision made no difference in wound pain or gastrointestinal recovery compared with totally LS. Otherwise, in open splenectomy, we have to retract the incisional wall the entire time, which appeared to result in greater 
postoperative wound or recovery problems. The conventional splenectomy procedure seriously affected patients' postoperative recovery. Hence, even if it is technically difficult, the laparoscopic approach should be chosen whenever possible, as it is associated with better outcomes. We could confirm the safety and effectiveness of LS in our study.

Although this study demonstrated good long-term follow-up results, lower morbidity and mortality rates, and a lower open conversion rate than other studies, it has some limitations, including the longer operation time and hospital stays. The mean operative time was 203 minutes (range, 115 475 minutes), while the median postoperative hospital stay was 8 days (range, $3 \sim 28$ days). Other studies reported operative times of $100 \sim$ 134 minutes, ${ }^{10,19-21}$ as well as hospital stays of $2.8 \sim 5.65$ days. ${ }^{19-21}$ This study had longer operation time than earlier studies owing to the delicate and careful handling during LS to minimize surgical complications and avoid open conversion in difficult cases that were indicated for LS. Further, this study had a relatively small group of 52 patients, which is lower than those of other studies.

Despite the disadvantages of LS, that is, longer operation time and intraoperative bleeding risk, especially in splenomegaly, if it is possible to ensure surgical stability, LS can be suggested earlier and more aggressively for treatment option with medical treatment.

\section{CONCLUSION}

In conclusion, LS is confirmed to be a safe and very effective procedure regardless of spleen size and disease. ${ }^{6}$ ITP is the most common indication for LS. In this study, the morbidity and mortality rates after LS were very low, even lower than those reported by previous studies. LS has very effective long term follow-up results, especially in ITP. Therefore, we should consider LS more actively for early treatment option in surgical disease of the spleen, such as ITP.

\section{REFERENCES}

1) Aydin C, Kayaalp C, Olmez A, Tatli F, Kirimlioglu V. Laparoscopic splenectomy with a vessel sealing device. Minim invasive Ther Allied Technol 2008;17:308-312.

2) Misawa $T$, Yoshida $K$, Iida $T$, et al. Minimizing intra-operative bleeding using a vessel sealing system and splenic hilum hanging maneuver in laparoscopic splenectomy. J Hepatobiliary Pancreat Surg 2009;16:786-791.

3) Romano F, Gelmini R, Caprotti R, et al. Laparoscopic splenectomy: LigaSure versus Endo GIA: a comparative study. J
Laparoendosc Adv Surg Tech A 2007;17:763-767.

4) Delaitre B, Champault G, Barrat C, et al. Laparoscopic splenectomy for haematologic disease: study of 275 cases. French Society of Laparoscopic Surgery. Ann Chir 2000;125: 522-529.

5) Beauchamp RD, Holzman MD, Fabian MPHTC, Weinberg JA. The spleen. In: Townsend CM Jr, Beauchamp RD, Evers BM, Mattox KL, editors. Sabiston Textbook of Surgery: The Biological Basis of Modern Surgical Practice. 18th ed. Philadelphia: Elsevier Saunders; 2008.

6) Richardson WS, Smith CD, Branum GC, Hunter JG. Leaning spleen: a new approach to laparoscopic splenectomy. J Am Coll Surg 1997;185:412-415.

7) Zheng CX, Zheng D, Chen LH, Yu JF, Wu ZM. Laparoscopic splenectomy for immune thrombocytopenic purpura at a teaching institution. Chin Med J (Engl) 2011;124:1175-1180.

8) Delaitre B, Maignien B. Splenectomy by the laparoscopic approach. Report of a case. Presse Med 1991;20:2263.

9) Winslow ER, Brunt LM. Perioperative outcome of laparoscopic versus open splenectomy: a meta-analysis with an emphasis on complication. Surgery 2003;134:647-653.

10) Machado NO, Al Kindy N, Chopra PJ. Laparoscopic splenectomy using LigaSure. JSLS 2010;14:547-552.

11) Vecchio R, Intagliata E, Cacciola E. Laparoscopic splenectomy: a single center experience. Updates Surg 2013 Aug 4 [Epub]. http://dx.doi.org/10.1007/s13304-013-0228-x.

12) Konkle B. Disorders of platelets and vessel wall. In: Charles W, Anthony F, Eugene B, Dennis K, Stephen H, editors. Harrison's Principles of Internal Medicine. 18th ed. New York: McGraw-Hill; 2011.p.968

13) Cohen YC, Djulbegovic B, Shamai-Lubovitz O, Mozes B. The bleeding risk and natural history of idiopathic thrombocytopenic purpura in patients with persistent low platelet counts. Arch Intern Med 2000;160:1630-1638.

14) McMillan R. Chronic idiopathic thrombocytopenic purpura. $\mathrm{N}$ Engl J Med 1981;304:1135-1147.

15) Cines DB, Blanchette VS. Immune thrombocytopenic purpura. N Engl J Med 2002;346:995-1008.

16) Bisharat N, Omari H, Lavi I, Raz R. Risk of infection and death among post-splenectomy patients. J Infect 2001;43:182186.

17) Vianelli N, Galli M, de Vivo A, et al. Efficacy and safety of splenectomy in immune thrombocytopenic purpura: long-term results of 402 cases. Haematologica 2005;90:72-77.

18) Kang CM, Lee JG, Kim KS, et al. Long-term follow-up of laparoscopic splenectomy in patients with immune thrombocytopenic purpura. J Korean Med Sci 2007;22:420-424.

19) Rosen M, Brody F, Walsh RM, Tarnoff M, Malm J, Ponsky J. Outcome of laparoscopic splenectomy based on hematologic indication. Surg Endosc 2002;16:272-279.

20) Pattenden CJ, Mann CD, Metcalfe MS, Dyer M, Lloyd DM. Laparoscopic splenectomy: a personal series of 140 consecu- 
tive cases. Ann R Coll Surg Engl 2010;92:398-402.

21) Bulus H, Mahmoud $H$, Altun $H$, Tas A, Karayalcin $K$. Outcomes of laparoscopic versus open splenectomy. J Korean Surg Soc 2013;84:38-42.
22) Swanson TW, Meneghetti AT, Sampath S, Connors JM, Panton ON. Hand-assisted laparoscopic splenectomy versus open splenectomy for massive splenomegaly: 20-year experience at a Canadian centre. Can J Surg 2011;54:189-193. 